\title{
THE EFFECT OF AQUEOUS ETHANOLIC EXTRACT OF ALCHORNEA CORDIFOLIA LEAF ON THE HISTOLOGY OF THE AORTA OF WISTAR RATS
}

\section{F. ELIAKIM-IKECHUKWU AND E. B. RIMAN}

\author{
Department of Anatomy, Faculty of Basic Medical Sciences, University of Calabar, Calabar, Nigeria \\ E-mail: chisom_favor@yahoo.com Tel: +234(0) 8032768463
}

Summary: Sixteen Wistar rats weighing 160-180g were used in this research work. The rats were divided into three groups, designated A, B, and C. Group A served as the control and consisted of four rats, whereas groups $\mathrm{B}$ and $\mathrm{C}$ consisted of six rats each and served as the experimental groups. A. cordifolia leaf extract was administered to the experimental groups $\mathrm{B}$ and $\mathrm{C}$ orally at a dose of $250 \mathrm{mg} / \mathrm{kg} / \mathrm{day}$ and $500 \mathrm{mg} / \mathrm{kg} / \mathrm{day}$ respectively. The control group received $0.5 \mathrm{ml}$ of normal saline. The administration lasted for fourteenth days. All the animals were given feed and water freely. The rats were sacrificed after the fourteenth day of administration of herbal extract and the aortae harvested and processed histologically using haematoxylin and eosin staining technique. Tissue sections revealed that $A$. cordifolia is capable of inducing elastogenesis in the aorta. This attribute of the herb may be beneficial in increasing elastic recoil of the aortic wall and may reduce blood pressure.

Keywords: Alchornea cordifolia, aorta, Wistar rats, Histology

\section{Introduction}

The history of the use of herbs in the management of diseases dates back to the time of the early man (Sofowora, 1982 and Kafara, 1994). In herbal medicine, herbs/plants are being used in their unaltered form for the treatment of disease. Alchornea cordifolia, a medium-sized shrubby tree is one of such herbs. It belongs to the subfamily Acalypholdeae and family Euphorbiaceae or Spurge family. The common names are 'Iporuru', 'Iporoni', 'Iporuro', Ipurosa', 'macochichua' and 'Nianda'. The English name is Christmas bush and the Efik name is 'Mbom'. It is widely distributed throughout Africa where it is used extensively in traditional medicine. It has been very valuable locally in some ethnic groups in Nigeria for the management of haemorrhoids and high blood pressure. It has been found to have antiinflammatory, antibacterial and analgesic properties (Cesario, 1993). The phytochemical include alchorneine, anthranilic acid, gentisinic acid, iso alchorneine, yohimbine and alkaloids (Duke and Vasquez, 1994).

Sub-Saharan Africa represents about $75 \%$ of the population that rely on herbal remedies. A majority of Nigerian population still rely on Traditional Medicine. Available evidence suggests that some herbal remedies and Traditional therapeutic regimes are efficacious and affordable (TMP, 2007).

Hypertension is a common health problem with sometimes devastating consequences. It is accompanied and influenced by significant changes in arterial function. Blood pressure is proportional to cardiac output and peripheral vascular resistance. Although changes in endothelial and smooth muscle cell function are important in acute control of total peripheral resistance and blood pressure, changes in blood vessel structure also contribute to long-term alterations in arterial (Carretero, 2005 and Jacob et al., 2001).

The aorta is the largest elastic artery in the body. The tunica media is rich in elastic fibres which are arranged in fairly compact layers separated by and alternating with layers of smooth muscle cells. The elastic components allow it to expand during systole, thereby storing some of the energy of each heart beat. Elastic recoil of the vascular wall propels blood through the peripheral vascular system (Kumar et al., 2005).

In early stages of hypertension, there is hypertrophy of the vascular wall with increase of smooth muscle cells and elastic fibres in the media. As hypertension progresses, this valuable protective adaptation is overcome and these hypertrophic changes give way to fibrous replacement of muscle and the elastic tissue may break up and undergo partial absorption and the wall becomes thickened and of increased rigidity (Anderson, 1985).

This study is aimed at determining if $A$. cordifolia will bring about a structural modification on the wall of aorta that may enhance its function..

\section{Materials and methods}

Sixteen Wistar rats weighing between 160 and $180 \mathrm{~g}$ were used in the study. They were housed in the animal house of department of Anatomy, University of Calabar, Calabar. The animals were fed with rat chow from Pfizer and given water freely. For the experiment, the animals were divided into three groups A, B, and C. Group A had four rats and served as the control and was given 
$0.5 \mathrm{ml}$ of normal saline while groups $\mathrm{B}$ and $\mathrm{C}$ had six rats each and received $250 \mathrm{mg} / \mathrm{kg} /$ day and $500 \mathrm{mg} / \mathrm{kg} /$ day of Alchornea cordifolia leaf extract respectively via orogastric intubation.

The tender part of the leaves were harvested, washed to remove dust particles and other debris. The leaves were air-dried in room temperature and thereafter blended into fine powdery form. Aqueous ethanolic extraction was done using the method described by Pieme et al., (2006). The filtrate got was allowed to dry. The herbal extract was administered in two divided doses daily for fourteen days. After this period of administration, the animals were anaesthetized using chloroform inhalation and a midline incision made to expose the thoracic cavity and the aorta harvested, washed in normal saline and then fixed in $10 \%$ neutral formalin. The tissues were then processed histologically using haematoxylin and eosin staining techniques for microscopy, (Lillie, 1954).

\section{Results}

Plate 1 - Group A (control) - Received $0.5 \mathrm{ml}$ of Normal saline.

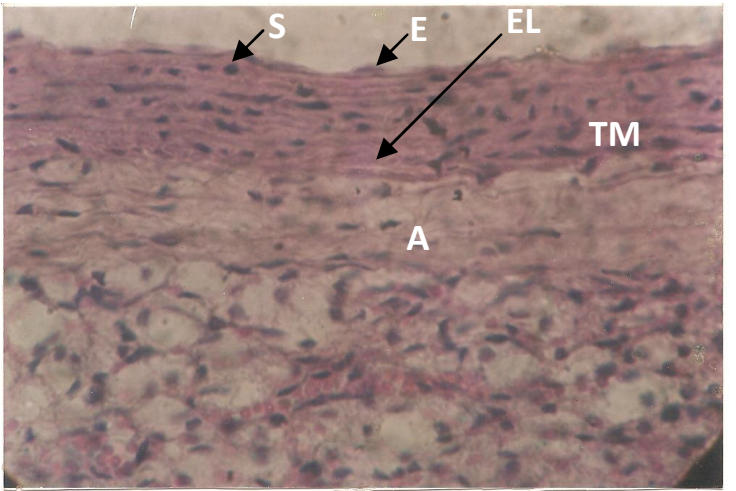

The tunica intima is seen with a conspicuous endothelial lining. The tunica media is seen made up of elastic fibres arranged like a set of membranes with some smooth muscles in between. E - Endothelium (Tunica intima), S - Smooth muscle cell, EL-Elastic fibre, TM - Tunica media, A - Tunica Adventitia; Magnification 150x

Plate 2 - Group B (Received 250mg/kg/day of Alchornea cordifolia leaf extract)

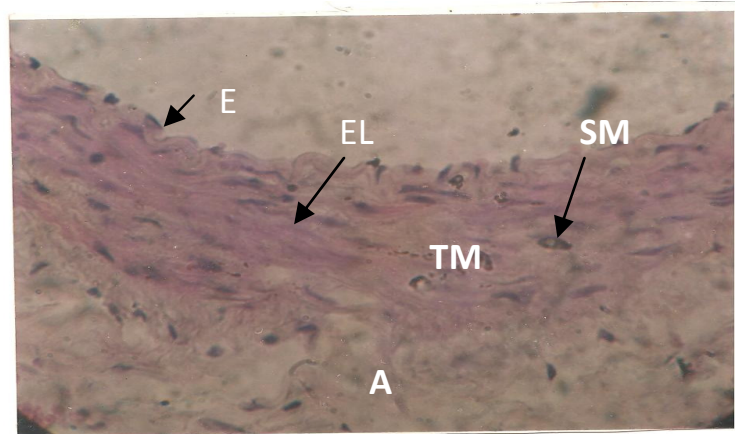

The tunica intima was normal. There was slight increase in elastic fibres in the tunica media thereby resulting to a slight increase in the thickness of aortic wall. The smooth muscle fibre did not seem to be affected but were slightly spaced apart as a result of laying down of new elastic fibres. Magnification 150x.

Plate 3 - Group C (Received 500mg/kg/day of Alchornea cordifolia leaf extract)

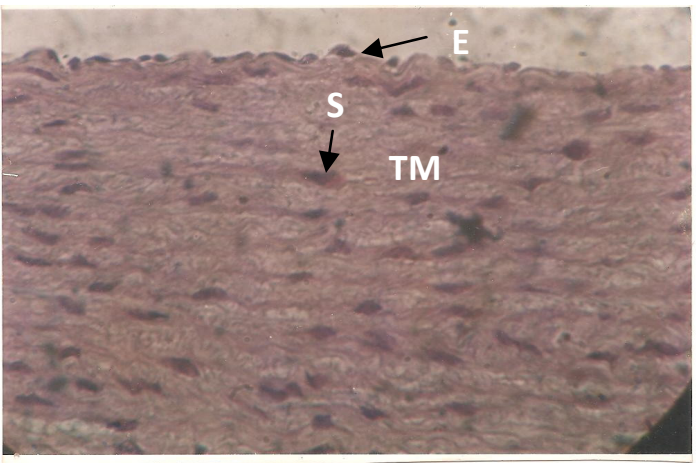

The tunica intima appears normal. In the tunica media there was marked increase in density of elastic fibres leading to appreciable increase in thickness or aortic wall. The smooth muscles were spaced farther apart but appeared to be slightly hypertrophied. Magnification 150x

\section{Discussion}

Active remodeling has been confirmed in the aorta and is observed as an increase in wall thickness and medial area. Arterial remodeling occurs in response to mechanical and neurohumoral stimuli. From it foregoing it is obvious that structural modification is possible in the aorta as a consequence of increased wall stress (Carretero, 2005 and Jacob et al., 2001). This structural adaptation to increased stress soon fails making the vessel rigid and vulnerable to rupture.

From the present study $A$. cordifolia at $500 \mathrm{mg} / \mathrm{kg} \mathrm{bwt} /$ day was found to have induced the laying down of elastic fibres which will eventually increase the elastic recoil of the aorta. It also caused some smooth muscle hypertrophy. Hyperplasia of the smooth muscle cells did not occur and no change was observed in the endothelial and adventitial layers. The mechanism behind this is not known since no work has been done in this direction. One mechanism important to arterial remodeling is the function of matrix metalloproteinases (MMP). These are proteases that allow for the dissolution and reorganization of extracellular matrix especially collagen and elastin. (Bobik and Tkdchuk, 2003; Nagase et al., 2006). The action of MMPs are offset and balanced by 
Alchornea cordifolia leaf aorta histology

tissue inhibitors of matrix metalloproteinase (TIMP). It is possible that $A$. cordifolia may have exerted this structural change in the tunica media through an interplay between the enzymes present in the arterial wall or through other mechanisms. Whatever the mechanism, the increase in elastic content of the tunica media of the aorta will contribute to long-term alterations in arterial function by smoothening out the intermittent pumping action of the heart under high pressure.

Further studies should be done in hypertensive animals to see how this works and also to establish the mechanisms behind this structural change.

\section{References}

Anderson, J. R. (1985). Muir's Textbook of Pathology. ELBS. 12th ed. pp.14-15.

Bobik, A. and T. Kaduk, V. (2003). Metallophrothinases \& plasminogen activators in vessel remodeling. Curr Hypteras Rep.5: 466-472.

Carretero, O. A. (2005) Vascular remodeling and the Kallikrein-kinin system. J. Clin. Invest 115:588-591.

Cesario, A. (1993). Screening of plants used in Argentine folk medicine for ntimicrobial activity. Journal of Ethnopharmacology 139:119-128.

Duke, J and Vasquez, R. (1994). Amazonian Ethnobotanical Dictionary, Florda, CRC Press Incorporated. 122:101-102.
Jacob, M. P.; Bracher-Commander, C.; Fontaine, V.; Benazzoug, Yeldman, L. and Michel, J. B. (2001). Extracellular matrix remodeling in the vascular wall. Pathol Biol. (Paris) 49:326-332.

Kafaru, E. (1994). Immense help from nature's workshop. Nigeria, Elikat Health Services Limited. p. 32.

Kumar, V.; Abbas, A. K. and Fausto, N. (2005). Robbins and Cotran Pathologic Basis of Disease, 7th ed. Elsevier Saunders. Pp. 512526.

Lillie, R. D. (1954). Histopathologic Technic and Practical Histochemistry. McGraw-Hill Company, Toronto. Pg. 114.

Nagase, H.; Visse, R. and Murphy, G. (2006). Structure and function of matrix metallophoteinases and TIMPs. Cardiovasc. Res 49:562-573.

Pieme, C. A.; Penlap, V. N.; Nkegoum, B., Taziebou, C. L.; Tekwu, E. M. and Ngongang (2006). Evaluation of acute and subacute toxicities of aqueous ethanolic extract of senna alata (L.) Roxl (ceasal piniaceae). Afric. J. Biotech. 5(3) 283-289.

TMP (2007). Traditional Medicine Policy for Nigeria. pp.66.

Received: November 20, 2009

Accepted: December 28, 2009 